\title{
PANORAMA DO DESIGN FOR ENVIRONMENT: UMA REVISÃO BIBLIOGRÁFICA SISTEMÁTICA
}

\section{PANORAMA OF DESIGN FOR ENVIRONMENT: A SYSTEMATIC LITERATURE REVIEW}

\author{
Marcos José Alves Pinto JuniorE-mail: marcos alvesir@yahoo.com.br \\ Juliana Veiga Mendes* E-mail: juveiga@ufscar.br \\ *Universidade Federal de São Carlos (UFSCar), Campus Sorocaba, São Paulo
}

\begin{abstract}
Resumo: Esta pesquisa é um estudo exploratório apresentando o Design for Environment - DfE como uma prática de gestão ambiental em Ecologia Industrial no ciclo de desenvolvimento do produto. O DfE analisa todas as etapas do ciclo de vida de um produto: desenvolvimento, fabricação, uso e disposição final propondo alterações no projeto, de forma a minimizar o impacto ambiental do produto. Com o intuito de analisar a literatura sobre o tema, esta pesquisa conduz uma Revisão Bibliográfica Sistemática - RBS sobre o DfE e práticas relacionadas. A RBS contempla uma etapa de planejamento, onde são definidos os objetivos da pesquisa, conceitos para inclusão e exclusão dos trabalhos a serem analisados, os trabalhos primários identificados e os materiais e métodos. A etapa seguinte é denominada processamento, que resulta mapeamento dos trabalhos. Nesta etapa, a análise dos objetivos dos artigos permitiu observar a convergência para temas relacionados à qualidade, inovação, estratégia, melhoria ambiental, avaliação ambiental, ferramenta, integração, setor ou produto específico estudado. A terceira etapa resultados, classifica os artigos por autoria e foco de pesquisa. Por fim esses artigos foram analisados e um conjunto de práticas relacionadas ao DfE disponíveis na literatura, foram classificadas de acordo com as etapas do ciclo de desenvolvimento do produto.
\end{abstract}

Palavras-chave: Gestão ambiental. RBS. DfE. Ciclo de desenvolvimento do produto. Práticas DfE.

Abstract: This research is an exploratory study presenting the Design for Environment - DfE as an environmental management practice in Industrial Ecology in the product development cycle. DfE analyzes all stages of the product life cycle: development, manufacturing, use and final disposal proposing changes in the design, to minimize the environmental impact of the product. To analyze the literature on the subject, this research conducts a Systematic Bibliographic Review - RBS on DfE and identifies related practices. The RBS performed can be divided into three stages: planning, processing, and results. In the planning stage, the objectives of the research are defined, concepts for inclusion and exclusion of the works to be analyzed, the identified original works and the materials and methods. The next step, processing, results in job mapping. In this stage, the analysis of the objectives of the articles allowed to observe the convergence for themes related to quality, innovation, strategy, environmental improvement, environmental assessment, tool, integration, sector or particular product studied. The third step, results, ranks the articles by authorship and research focus. Finally, these articles were analyzed, and a set of DfE-related practices available in the literature was classified according to the stages of the product development cycle.

Keywords: Environmental management. RBS. DfE. Product development cycle. DfE practices.

\section{INTRODUÇÃO}

A aplicação de práticas ambientais e estratégias que previnem ou minimizam

Revista Produção Online. Florianópolis, SC, v. 18, n. 1, p. 310-328, 2018. 
danos ao meio ambiente gerados por processos industriais, estão se tornando fundamentais (GIANNETTI; ALMEIDA, 2006) para uma empresa se ajustar e permanecer no mercado.

Práticas de gestão ambiental são programas de melhoria do desempenho ambiental de processos e produtos (MATOS; HALL, 2007; MIETTINEM; HAMALAINEN, 1997; MONTABON, SROUFE, NARASIMHAN, 2007; SROUFE, 2003). Podem ajudar no controle do uso de recursos durante o processo produtivo ou também no projeto do produto (ANDRIANKAJA et al., 2015; HOUE; GRABOT, 2009; ZHANG et al., 2011; CHIANG; ROY, 2012; FIKSEL, 2009). A integração de aspectos ambientais em produtos pode minimizar os riscos de emissões difundidas durante a produção, consumo e descarte (BAUMANN; BOONS; BRAGD, 2002; DANGELICO; PONTRANDOLFO, 2010).

O Design for Environment - DfE é uma prática de projeto de produto baseada na minimização de consequências negativas no âmbito econômico, ambiental e social, em questões durante e além do ciclo de vida dos mesmos (CHARTER; TISCHNER, 2001; FIKSEL, 1997). Diferente de outras práticas de gestão ambiental, o DfE analisa todas as fases do ciclo de desenvolvimento do produto com o objetivo de minimizar o impacto ambiental (CHARTER; TISCHNER, 2001; FIKSEL, 1997; ZHANG et al., 2011; CHIANG; ROY, 2012). Também, como aplicado no projeto de produto, se diferencia de projetos de produto tradicionais, onde são produtivistas e não consideram o impacto no meio ambiente (GIANNETTI; ALMEIDA, 2006).

Com a importância das considerações sobre o impacto ambiental dos produtos em geral, esta pesquisa faz uma Revisão Bibliográfica Sistemática - RBS para o DfE e tem por objetivo analisar a literatura relacionada e circunstanciá-la no âmbito do ciclo de vida. O propósito da análise consiste em classificar as práticas de DfE, identificadas na revisão bibliográfica, no contexto das etapas do ciclo de desenvolvimento do produto, evidenciando assim, sua abrangência e ampla utilização. A RBS é inspirada no roteiro de RBS de Conforto, Amaral e Silva (2011).

Este trabalho está fundamentado em torno de uma questão central, representando o núcleo da investigação e utilizando conceitos e termos específicos, abordados para a informação relacionada, pré-definida, focada e estruturada em uma questão específica (BIOLCHINI et al., 2005). 
Inicialmente, foi realizada a leitura de materiais sendo referencial teórico primário. Não foi encontrado nenhum trabalho sistematizando o DfE em um conjunto de práticas. Desta forma, a RBS analisa os artigos em bases científicas, identificando características e aplicação da forma de estudo do DfE, como também as práticas mais utilizadas.

Esse artigo está estruturado da seguinte forma: após essa introdução ao assunto, a seção 2 detalha o método de pesquisa. Na seção 3 os resultados das etapas da RBS são apresentados e, por fim, as discussões e considerações finais, na seção 4.

\section{MÉTODOS}

Para a realização da RBS e posterior análise dos trabalhos, os procedimentos metodológicos utilizados neste estudo exploratório estão representados na Figura 1.

Figura 1 - Modelo para condução da RBS
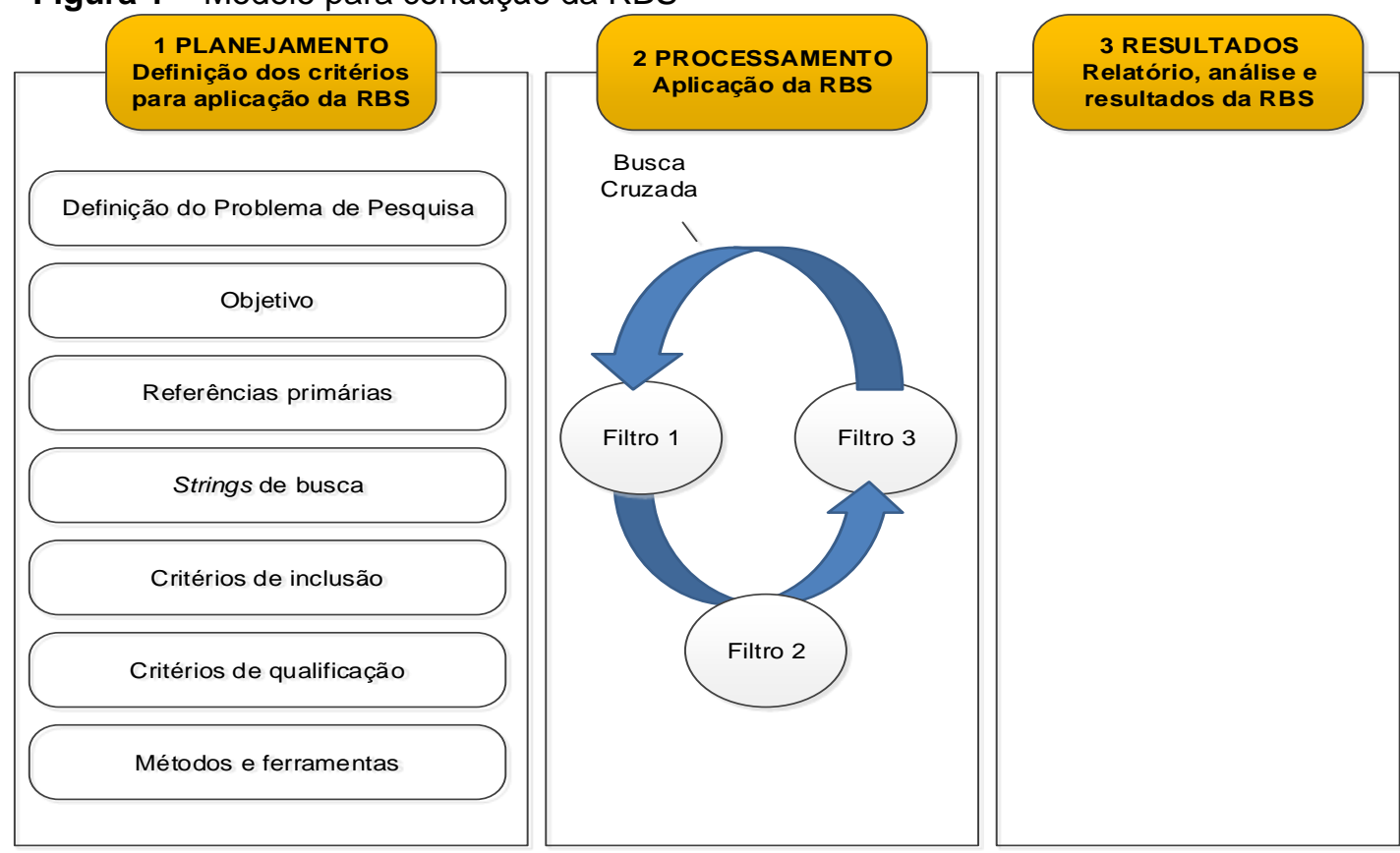

Fonte: Adaptado de Conforto, Amaral e Silva (2011)

O modelo adotado para a condução da RBS possui 3 etapas principais, a saber: planejamento, processamento e resultados. O planejamento é constituído por sete passos os quais são fundamentais para um bom resultado, sendo metódicos, transparentes e replicáveis (COOK et al., 1997; COOPER, 1998). Na etapa de 
processamento os artigos pré-selecionados foram analisados considerando três critérios de seleção (ou filtros), aplicados consecutivamente. E por fim a terceira etapa, onde os trabalhos selecionados são analisados e categorizados.

A seção a seguir apresenta os resultados da RBS.

\section{CONDUÇÃO DA RBS}

A condução da RBS é explicada detalhadamente nos tópicos a seguir e foi organizada seguindo o modelo apresentado na figura 1.

\subsection{Etapa 1 - Planejamento da RBS}

A primeira ação, na etapa de planejamento, consiste na obtenção de referências primárias para o tema DfE. Estas referências foram pesquisadas nas bases de periódicos Science Direct e ISI Web of Knowledge sendo buscas realizadas somente pela palavra-chave DfE, da Universidade Federal de São Carlos.

Posteriormente, foram identificadas, nos artigos primários, palavras-chave para construção das strings de busca nas bases de periódicos. As bases de periódicos apresentam características de pesquisa e apresentação de buscas de forma diferente entre si, por isso ambas estão detalhadas a seguir. Porém, este fator de diferenciação de bases dos periódicos não teve impacto nas palavras-chave quanto ao tema pesquisado.

Os critérios de inclusão para etapa de processamento, com intuito de selecionar os artigos utilizados para a etapa de saída são:

- Utilização de artigos disponíveis de forma gratuita;

- $\quad$ Seleção dos artigos que contenham no título as strings definidas;

- Utilização dos artigos disponíveis nas áreas de "Engineering", "Environmental Sciences Ecology" e "Operations Research Management Science".

- $\quad$ Artigos disponíveis em língua portuguesa ou inglesa;

- Utilizados somente artigos.

Os critérios de qualificação dos trabalhos analisados foram definidos para avaliar a importância do artigo para este estudo, sendo observados a abordagem, o 
método de investigação utilizado, objetivo geral definido, área de pesquisa do trabalho e ramo ou setor estudado.

As buscas foram realizadas nos títulos dos trabalhos por meio das strings definidas. Assim, critérios de inclusão para seleção foram executados, sendo a área de pesquisa selecionada, os artigos disponíveis gratuitamente para download e verificação dos artigos em duplicata. Os endereços eletrônicos das bases de dados para esta pesquisa e um resumo da etapa de planejamento, estão disponíveis no Quadro 1. Para armazenamento das informações resultantes deste processo foram os softwares Microsoft Excel (Filtro 1) e o JabRef Reference Manager 2.10 (Filtros 2 e 3).

Quadro 1 - Resumo da etapa de planejamento da RBS

\begin{tabular}{|c|l|}
\hline Referências Primárias & $\begin{array}{l}\text { Bevilacqua, Ciarapica e Giacchetta (2012); Birch, Hon e Short } \\
\text { (2012); Borchardt et al. (2012); Calcott e Walls (2005); Fiksel } \\
\text { (2009); Lindahl (2006); Zhang et al. (2011). }\end{array}$ \\
\hline Palavras-chave & $\begin{array}{l}\text { Design for Environment; design for the environment; ecodesign; } \\
\text { projeto para o meio ambiente. }\end{array}$ \\
\hline $\begin{array}{c}\text { Strings de busca: } \\
\text { ISI Web of Knowledge }\end{array}$ & $\begin{array}{l}\text { ("design for environment") OR ("design for the environment") OR } \\
\text { ("ecodesign") }\end{array}$ \\
\hline $\begin{array}{c}\text { Strings de busca: } \\
\text { Scopus }\end{array}$ & $\begin{array}{l}\text { ( TITLE ( "design for environment" ) OR TITLE ( "design for the } \\
\text { environment" ) OR TITLE ( "ecodesign" ) OR TITLE ( "projeto } \\
\text { para o meio ambiente" ) ) }\end{array}$ \\
\hline $\begin{array}{c}\text { Endereço eletrônico: } \\
\text { ISI Web of Knowledge }\end{array}$ & $\begin{array}{l}\text { http://apps.webofknowledge.com/UA_GeneralSearch_input.do?pro } \\
\text { duct=UA\&search_mode=GeneralSearch\&SID=2ErSOGYIsUhYmB } \\
\text { NUC3C\&preferencesSaved= }\end{array}$ \\
\hline Endereço eletrônico: & http://www.scopus.com \\
\hline Scopus &
\end{tabular}

Fonte: Elaborado pelos autores

\subsection{Etapa 2 - Processamento da RBS}

$\mathrm{Na}$ etapa de processamento foram aplicados 3 filtros explicados a seguir. $\mathrm{O}$ processamento é executado utilizando as strings definidas para busca nas bases de dados. Os filtros têm por objetivo selecionar os trabalhos que se relacionam com o objetivo central da pesquisa em sua leitura.

O filtro 1 é definido para leitura do título, resumo e palavras-chave dos trabalhos encontrados na busca das bases de dados. É apresentada esta etapa na Tabela 1, atendendo os critérios de inclusão. 
Tabela 1 - Resultado das etapas de buscas

\begin{tabular}{lccc}
\multicolumn{1}{c}{ Detalhamento de busca } & ISI WoK & Scopus & Total \\
\hline Total bruto & 431 & 468 & 899 \\
Seleção área pesquisada & 357 & 391 & 748 \\
Tipo de documento (artigo) & 110 & 157 & 267 \\
Idioma & 103 & 151 & 254 \\
Artigos disponíveis gratuitamente & 59 & 122 & 181 \\
Eliminação de duplicatas & & 127 & \\
\hline
\end{tabular}

Fonte: Elaborado pelos autores

O total bruto de 899 representa todos os trabalhos encontrados nas bases de dados com a utilização das strings. Após a aplicação dos critérios de inclusão restaram 181 trabalhos. Como são duas bases de dados foram excluídos os trabalhos duplicados, sendo 127 artigos selecionados.

Para o filtro 2 foi realizado a leitura da introdução e conclusão dos 127 artigos selecionados no filtro 1. Após registros do objetivo geral dos trabalhos e seu método de investigação, foram selecionados 82 artigos que foram submetidos ao filtro 3 . Os objetivos dos trabalhos e métodos dos trabalhos foram relevantes para seleção, cumprindo o objetivo desta pesquisa.

O filtro 3 requer leitura completa dos trabalhos para elaboração dos fichamentos, sendo selecionados 41 artigos. Adicional a este filtro para posterior elaboração da saída deste protocolo, foram adicionados 2 livros pois são referências relevantes identificadas nas análises realizadas.

O resumo dos critérios de leitura para seleção dos filtros é representado na Figura 2.

Figura 2 - Critérios de leitura para seleção dos Filtros
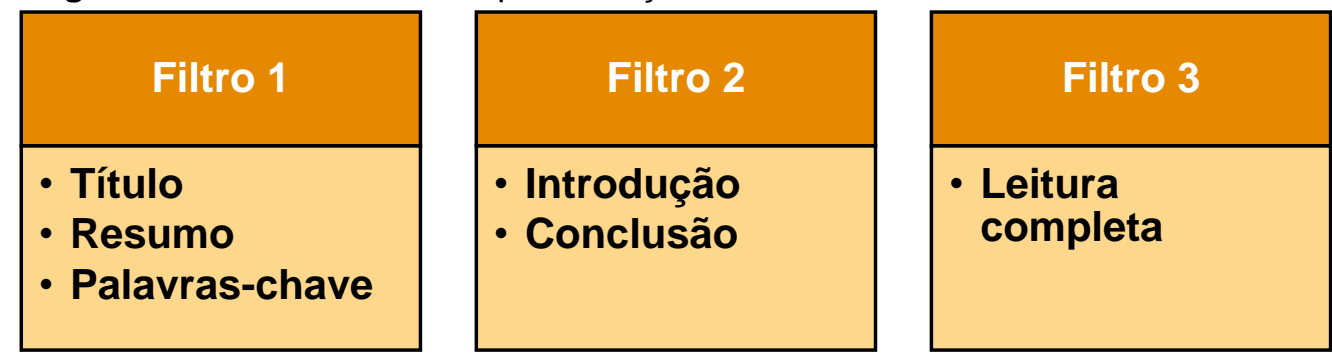

Fonte: Elaborado pelos autores 


\subsection{Etapa 3 - Resultados da RBS}

No Quadro 2 são apresentados os resultados principais da RBS.

Quadro 2 - Resumo da RBS

\begin{tabular}{|c|c|}
\hline Base de dados & ISI Web of Knowledge; Scopus \\
\hline $\begin{array}{c}\text { Total bruto de artigos } \\
\text { encontrados }\end{array}$ & 899 \\
\hline $\begin{array}{c}\text { Total dos artigos } \\
\text { selecionados }\end{array}$ & 21 \\
\hline $\begin{array}{c}\text { Referências } \\
\text { cruzadas }\end{array}$ & 2 livros \\
\hline Palavras-chave & $\begin{array}{c}\text { Design for Environment; design for the } \\
\text { environment; ecodesign; projeto para o } \\
\text { meio ambiente }\end{array}$ \\
\hline
\end{tabular}

Fonte: Elaborado pelos autores

Referente ao total bruto encontrado nas bases de dados foi elaborado um gráfico de evolução temporal do campo de pesquisa das bases separadamente, de forma a quantificar os trabalhos publicados e entender o interesse pelo assunto tratado nesta pesquisa, apresentados nas Figuras 3 e 4.

Observa-se que o total dos trabalhos encontrados sobre o assunto DfE nas bases de dados são bem próximos. São 431 trabalhos encontrados na base ISI Web of Knowledge e 468 na base Scopus. A visualização gráfica (Figuras 3 e 4) permite apontar o aumento de interesse pelo tema, a partir de 1994.

Figura 3 - Evolução DfE- base Web Knowledge

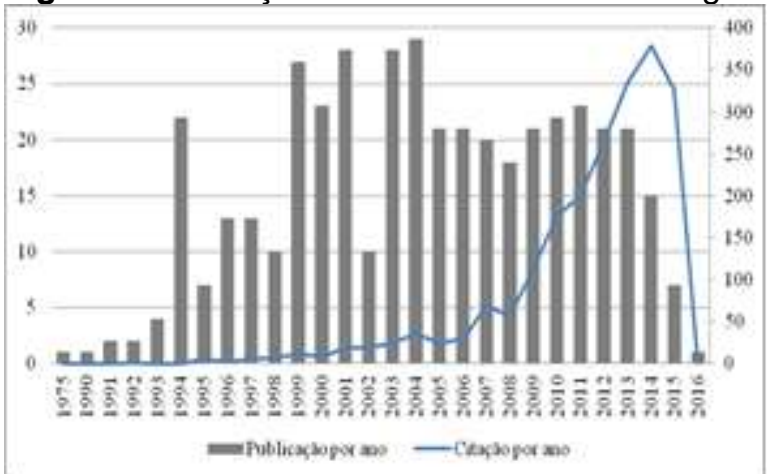

Fonte: Elaborado pelos autores
Figura 4 - Evolução DfE - base Scopus

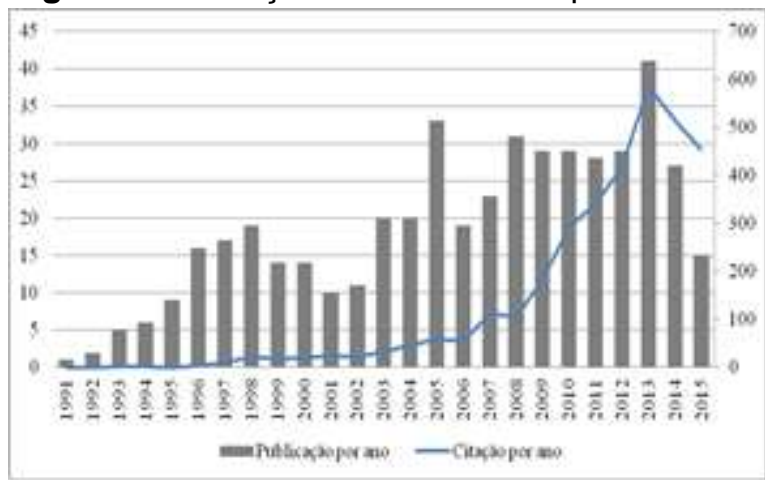

Fonte: Elaborado pelos autores 
Uma síntese dos trabalhos selecionados nas bases de dados é apresentado no Quadro 3. Cada trabalho foi identificado pela autoria, método de investigação, abordagem, setor estudado e forma de contribuição. Os trabalhos foram pesquisados nas bases de dados definidas nesta pesquisa. Na Tabela 2 é apresentada uma equivalência relativa por periódico em porcentagem. Também é mencionado o Qualis Capes (Engenharias III, área de pesquisa deste trabalho) e o Fator de Impacto de cada periódico.

Observa-se uma concentração de artigos sobre o tema no Journal of Cleaner Production, representando $66 \%$ de todos os trabalhos selecionados.A análise nos artigos obtidos com a RBS, permitiu identificar práticas relacionadas ao DfE que foram classificadas considerando as etapas do ciclo de desenvolvimento do produto (Lewis et al., 2001): desenvolvimento ou concepção, produção ou manufatura, uso ou utilização e disposição ou recuperação (Quadro 4).

Tabela 2 - Estratificação por periódicos dos trabalhos para RBS

\begin{tabular}{|c|c|c|c|c|}
\hline Periódicos & Quantidade & $\begin{array}{l}\text { \% Relativa } \\
\text { Quantidade }\end{array}$ & Qualis Capes & Fator de Impacto \\
\hline Journal of Cleaner Production & 27 & $66 \%$ & A 1 & 3.844 \\
\hline Science of the Total Environment & 1 & $2 \%$ & A 1 & 4.099 \\
\hline Expert Systems with Applications & 2 & $5 \%$ & A 2 & 2.240 \\
\hline Resources, Conservation and Recycling & 2 & $5 \%$ & A 2 & 2.564 \\
\hline Engenharia Sanitária e Ambiental & 1 & $2 \%$ & B2 & 0.241 \\
\hline Produção & 2 & $5 \%$ & B2 & 0.230 \\
\hline Ambiente \& Sociedade & 1 & $2 \%$ & B3 & 0.049 \\
\hline
\end{tabular}

Fonte: Elaborado pelos autores 
Quadro 3 - Seleção dos trabalhos para RBS

\begin{tabular}{|c|c|c|c|c|}
\hline Autores & $\begin{array}{l}\text { Método de } \\
\text { Investigação }\end{array}$ & Abordagem & Setor /Produto & Contribuição \\
\hline Alves et al. (2010) & Estudo de caso & Qualitativa & Automobilística & Prática aplicável \\
\hline Andriankaja et al. (2015) & Estudo de caso & Qualitativa & Transportes & Framework teórico \\
\hline $\begin{array}{l}\text { Arana-Landin e Heras-Saizarbitoria } \\
\text { (2011) }\end{array}$ & Estudo de caso & Qualitativa & Não especificado & Framework teórico \\
\hline Ardente, Mathieux e Recchioni (2014) & Estudo de caso & Qualitativa & Eletrônicos & Narrativa conceitual \\
\hline Birch, Hon e Short (2012) & Experimentação & Qualitativa & Não especificado & Framework teórico \\
\hline Boks (2006) & Survey & Qualitativa & Não especificado & Framework teórico \\
\hline Boks e Stevels (2007) & Estudo de caso & Qualitativa & Indústria de eletrônicos & Prática aplicável \\
\hline Bonilla et al. (2010) & Estudo de caso & $\begin{array}{l}\text { Qualitativa } \\
\text { Quantitativa }\end{array}$ & Bebidas & Ferramenta \\
\hline Bonvoisin et al. (2014) & Estudo de caso & Qualitativa & Servicos & Framework teórico \\
\hline Borchardt et al. (2008) & Estudo de caso & Qualitativa & Automobilística & Prática aplicável \\
\hline Borchardt et al. (2010) & Estudo de caso & Qualitativa & Manufatura calçadista & Prática aplicável \\
\hline Borchardt et al. (2012) & $\begin{array}{l}\text { Estudo de caso } \\
\text { Modelagem }\end{array}$ & $\begin{array}{l}\text { Qualitativa } \\
\text { Quantitativa }\end{array}$ & Indústria química & $\begin{array}{l}\text { Avaliação da presença } \\
\text { do DfE }\end{array}$ \\
\hline Bovea e Pérez-Belis (2012) & Teórico & Qualitativa & Publicações & Tabela conceitual \\
\hline Brones e de Carvalho (2015) & Teórico & Qualitativa & Publicações & Framework teórico \\
\hline Brones, de Carvalho e Zancul (2014) & Estudo de caso & Qualitativa & $\begin{array}{l}\text { Fabricante bens } \\
\text { de consumo }\end{array}$ & Framework teórico \\
\hline Byggeth e Hochschorner (2006) & Teórico & Qualitativa & Não especificado & $\begin{array}{l}\text { Aplicação de } \\
\text { ferramentas }\end{array}$ \\
\hline González-García et al. (2011) & Estudo de caso & Quantitativa & Móveis & Tabela conceitual \\
\hline $\begin{array}{l}\text { Gouda, Jonnalagedda, Saranga } \\
\text { (2016) }\end{array}$ & Modelagem & Quantitativa & Automobilística & Modelo matemático \\
\hline Hernandez et al. (2012) & Teórico & Qualitativa & Não especificado & Ferramenta \\
\hline Jeswiet e Hauschild (2005) & Teórico & Qualitativa & Manufatura & Análise temporal \\
\hline Kengpol e Boonkanit (2011) & Teórico & $\begin{array}{c}\text { Qualitativa } \\
\text { Quantitativa }\end{array}$ & Não especificado & Framework teórico \\
\hline Kurk e Eagan (2008) & Teórico & Qualitativa & Não especificado & Framework teórico \\
\hline Lindahl (2006) & Estudo de caso & Qualitativa & $\begin{array}{l}\text { Equipamentos } \\
\text { industriais }\end{array}$ & Framework teórico \\
\hline Lofthouse (2006) & Estudo de caso & Qualitativa & Eletrodoméstico & Framework teórico \\
\hline Luttropp e Lagerstedt (2006) & Teórico & Qualitativa & Publicações & Check list \\
\hline Park e Tahara (2008) & Estudo de caso & $\begin{array}{l}\text { Qualitativa } \\
\text { Quantitativa }\end{array}$ & Câmera digital & Prática aplicável \\
\hline Passarini et al. (2010) & Experimentação & Quantitaitva & Automobilística & Prática aplicável \\
\hline Pigosso et al. (2010) & Teórico & Qualitativa & Não especificado & Análise de casos \\
\hline $\begin{array}{l}\text { Pigosso, Rozenfeld e McAloone } \\
\text { (2013) }\end{array}$ & $\begin{array}{l}\text { Hipotético- } \\
\text { dedutivo }\end{array}$ & Qualitativa & Manufatura & Framework teórico \\
\hline Platcheck et al. (2008)a & Estudo de caso & Qualitativa & Eletrônicos & Framework teórico \\
\hline Platcheck et al. (2008)b & Estudo de caso & Qualitativa & Compressor de ar & Prática aplicável \\
\hline Plouffe et al. (2011) & Survey & Quantitativa & $\begin{array}{l}\text { Manufatureiro e } \\
\text { serviços }\end{array}$ & Análise econômica \\
\hline Pochat, Bertoluci e Froelich (2007) & Estudo de caso & Qualitativa & Manufatura & Framework teórico \\
\hline Raz, Druehl e Blass (2013) & Modelagem & Quantitativa & Jornais & Modelo matemático \\
\hline Roca et al. (2012) & Lógica fuzzy & Quantitativa & Garrafa & Prática aplicável \\
\hline Sakundarini et al. (2015) & Estudo de caso & Qualitativa & Indústrias & Framework teórico \\
\hline Short et al. (2012) & Estudo de caso & $\begin{array}{l}\text { Qualitativa } \\
\text { Quantitativa }\end{array}$ & Manufatura & Análise de casos \\
\hline Silva, Moraes e Machado (2015) & Estudo de caso & Qualitativa & $\begin{array}{c}\text { Acessórios para } \\
\text { motocicleta }\end{array}$ & Framework teórico \\
\hline Tiruta-Barna et al. (2014) & Estudo de caso & Quantitativa & $\begin{array}{c}\text { Estação de tratamento } \\
\text { de água }\end{array}$ & Prática aplicável \\
\hline van Hemel e Cramer (2002) & Estudo de caso & Qualitativa & PME's & Framework teórico \\
\hline Zhang et al. (2011) & Estudo de caso & $\begin{array}{c}\text { Qualitativa } \\
\text { Quantitativa }\end{array}$ & Fabricante leite soja & Framework teórico \\
\hline
\end{tabular}

Fonte: Elaborado pelos autores 
Quadro 4 - Práticas relacionadas ao DfE categorizadas no ciclo de desenvolvimento do produto

(continua)

\begin{tabular}{|c|c|c|}
\hline Fase & $\begin{array}{l}\text { Práticas de DfE } \\
\end{array}$ & Literatura \\
\hline \multirow{19}{*}{$\begin{array}{l}\text { Desenvolvimento/ } \\
\text { Concepção }\end{array}$} & $\begin{array}{l}\text { Reduzir ou eliminar o uso de materiais ligados à } \\
\text { degradação da camada de ozônio e às mudanças } \\
\text { climáticas, durante o ciclo de vida }\end{array}$ & $\begin{array}{l}\text { Giannetti e Almeida (2006); González-García et al. } \\
\text { (2011); Borchardt et al. (2008); Short et al. (2012). }\end{array}$ \\
\hline & $\begin{array}{l}\text { Entender o produto como ambientalmente correto } \\
\text { por todo o seu ciclo de vida }\end{array}$ & $\begin{array}{l}\text { Giannetti e Almeida (2006); Bovea e Pérez-Bélis } \\
\text { (2012); Roca et al. (2012); van Hemel e Cramer } \\
\text { (2002). }\end{array}$ \\
\hline & $\begin{array}{l}\text { Escolher os materiais mais adequados, naturais } \\
\text { ou não, com base na avaliação do ciclo de vida }\end{array}$ & $\begin{array}{l}\text { Giannetti e Almeida (2006); González-García et al. } \\
\text { (2011); Passarini et al. (2010). }\end{array}$ \\
\hline & $\begin{array}{l}\text { Minimizar o consumo de energia, maximizando o } \\
\text { uso de fontes renováveis de energia }\end{array}$ & $\begin{array}{l}\text { Giannetti e Almeida (2006); Luttropp e Lagerstedt } \\
\text { (2006); Passarini et al. (2010); van Hemel e Cramer } \\
\text { (2002). }\end{array}$ \\
\hline & $\begin{array}{l}\text { Usar o mínimo de material e evitar a utilização de } \\
\text { materiais escassos }\end{array}$ & Giannetti e Almeida (2006). \\
\hline & $\begin{array}{l}\text { Reduzir ou eliminar o uso de materiais tóxicos, } \\
\text { inflamáveis ou explosivos durante o ciclo de vida }\end{array}$ & $\begin{array}{l}\text { Giannetti e Almeida (2006); Luttropp e Lagerstedt } \\
\text { (2006); Borchardt et al. (2008); Jeswiet e Hauschild } \\
\text { (2005); Boks (2006); Park e Tahara (2008); Ardente, } \\
\text { Mathieux e Recchioni (2014); Kurk e Eagan (2008). }\end{array}$ \\
\hline & Atender às regulamentações & Giannetti e Almeida (2006). \\
\hline & $\begin{array}{l}\text { Usar materiais de alta qualidade para minimizar o } \\
\text { peso do produto, se tais escolhas não interferem } \\
\text { com a necessária flexibilidade, resistência ao } \\
\text { impacto ou outras prioridades funcionais }\end{array}$ & $\begin{array}{l}\text { Wimmer et al. (2005); Luttropp e Lagerstedt (2006); } \\
\text { Fiksel (2009); van Hemel e Cramer (2002); Alves et al. } \\
\text { (2010); Park e Tahara (2008). }\end{array}$ \\
\hline & $\begin{array}{l}\text { Investir em melhores materiais, tratamentos de } \\
\text { superfície ou arranjos estruturais para proteger os } \\
\text { produtos de sujeira, corrosão e desgaste, } \\
\text { garantindo assim a manutenção reduzida e maior } \\
\text { vida útil do produto }\end{array}$ & Luttropp e Lagerstedt (2006). \\
\hline & $\begin{array}{l}\text { Usar o mínimo de elementos de união possível } \\
\text { (utilizar parafusos, adesivos, soldadura, encaixe } \\
\text { ajustável, fecho geométrico) }\end{array}$ & Luttropp e Lagerstedt (2006). \\
\hline & $\begin{array}{l}\text { Utilizar ferramentas disponíveis ou de fácil acesso } \\
\text { para o DfE }\end{array}$ & $\begin{array}{l}\text { Andriankaja et al. (2015); Passarini et al. (2010); } \\
\text { Hernandez et al. (2012); Luttropp e Lagerstedt (2006); } \\
\text { Lofthouse (2006); Lindahl (2006); Tiruta-Barna et al. } \\
\text { (2014); Byggeth e Hochschorner (2006); Birch, Hon e } \\
\text { Short et al. (2012); Kurk e Eagan (2008). }\end{array}$ \\
\hline & $\begin{array}{l}\text { Integrar o processo de DfE na cadeia de valor da } \\
\text { empresa }\end{array}$ & $\begin{array}{l}\text { Andriankaja et al. (2015); Bovea e Pérez-Bélis (2012); } \\
\text { Brones e de Carvalho (2015); Pochat, Bertoluci e } \\
\text { Froelich (2007); Boks (2006). }\end{array}$ \\
\hline & $\begin{array}{l}\text { Identificar prazos para o cumprimento das práticas } \\
\text { de DfE na fase de concepção }\end{array}$ & Andriankaja et al. (2015). \\
\hline & $\begin{array}{l}\text { Garantir confiabilidade dos resultados alcançados } \\
\text { do projeto do produto }\end{array}$ & Andriankaja et al. (2015). \\
\hline & $\begin{array}{l}\text { Obter um processo integrado às exigências } \\
\text { ambientais legais com o processo de DfE, } \\
\text { contemplando aspectos de segurança, } \\
\text { econômicos, jurídicos e funcionais }\end{array}$ & $\begin{array}{l}\text { Bovea e Pérez-Bélis (2012); Luttropp e Lagerstedt } \\
\text { (2006); Brones, de Carvalho e Zancul (2014); Brones } \\
\text { e de Carvalho (2015); Pochat, Bertoluci e Froelich } \\
\text { (2007); Arana-Landin e Heras-Saizarbitoria (2011); } \\
\text { Boks (2006). }\end{array}$ \\
\hline & $\begin{array}{l}\text { Identificar os requisitos necessários na fase de } \\
\text { concepção relacionados as exigências de } \\
\text { segurança }\end{array}$ & $\begin{array}{l}\text { Bovea e Pérez-Bélis (2012); Roca et al. (2012); Raz, } \\
\text { Druehl e Blass (2013); Jeswiet e Hauschild (2005); } \\
\text { Luttropp e Lagerstedt (2006). }\end{array}$ \\
\hline & $\begin{array}{l}\text { Identificar os } \\
\begin{array}{l}\text { requisitos necessários na fase de } \\
\text { concepção } \\
\text { econômicas }\end{array}\end{array}$ & $\begin{array}{l}\text { Bovea e Pérez-Bélis (2012); Roca et al. (2012); Raz, } \\
\text { Druehl e Blass (2013); Jeswiet e Hauschild (2005); } \\
\text { Luttropp e Lagerstedt (2006); Pigosso et al. (2010); } \\
\text { Plouffe et al. (2011). }\end{array}$ \\
\hline & $\begin{array}{l}\text { Identificar os requisitos necessários na fase de } \\
\text { concepção relacionados as exigências funcionais }\end{array}$ & $\begin{array}{l}\text { Bovea e Pérez-Bélis (2012); Roca et al. (2012); } \\
\text { Passarini et al. (2010); Raz, Druehl e Blass (2013); } \\
\text { Jeswiet e Hauschild (2005); Luttropp e Lagerstedt } \\
\text { (2006); Pigosso et al. (2010). }\end{array}$ \\
\hline & $\begin{array}{l}\text { Redução dos custos e impactos ambientais } \\
\text { considerando a análise do ciclo de vida }\end{array}$ & $\begin{array}{l}\text { Bonvoisin et al. (2014); González-García et al. (2011); } \\
\text { Raz, Druehl e Blass (2013); Park e Tahara (2008); } \\
\text { Kurk e Eagan (2008). }\end{array}$ \\
\hline
\end{tabular}

Revista Produção Online. Florianópolis, SC, v. 18, n. 1, p. 310-328, 2018. 
Quadro 4 - Práticas relacionadas ao DfE categorizadas no ciclo de desenvolvimento do produto

(continução)

\begin{tabular}{|c|c|c|}
\hline Fase & Práticas de DfE & \begin{tabular}{|c|} 
Literatura \\
\end{tabular} \\
\hline \multirow{7}{*}{$\begin{array}{l}\text { Desenvolvimento/ } \\
\text { Concepção }\end{array}$} & Utilizar de ferramentas DfE simples & $\begin{array}{l}\text { Bovea e Pérez-Bélis (2012); Hernandez et al. (2012); } \\
\text { Luttropp e Lagerstedt (2006); Lofthouse (2006); } \\
\text { Lindahl (2006); Byggeth e Hochschorner (2006); Birch, } \\
\text { Hon e Short et al. (2012); Kurk e Eagan (2008). }\end{array}$ \\
\hline & $\begin{array}{l}\text { Identificar os requisitos necessários na fase de } \\
\text { concepção quanto as exigências jurídicas/legais }\end{array}$ & $\begin{array}{l}\text { Bovea e Pérez-Bélis (2012); Roca et al. (2012); } \\
\text { Passarini et al. (2010); Sakundarini et al. (2015); } \\
\text { Gouda, Jonnalagedda, Saranga (2016); Jeswiet e } \\
\text { Hauschild (2005); Luttropp e Lagerstedt (2006); } \\
\text { Pigosso, Rozenfeld e McAloone (2013); Pigosso et } \\
\text { al. (2010); Ardente, Mathieux e Recchioni (2014). }\end{array}$ \\
\hline & Definir critérios para definição das informações & Bonvoisin et al. (2014). \\
\hline & $\begin{array}{l}\text { Empregar um modo de transporte com energia } \\
\text { eficiente }\end{array}$ & van Hemel e Cramer (2002). \\
\hline & $\begin{array}{l}\text { Identificar e aplicar os requisitos de clientes para o } \\
\text { produto e processo }\end{array}$ & Zhang et al. (2011). \\
\hline & $\begin{array}{l}\text { Assegurar o compromisso, apoio e recursos para } \\
\text { executar atividades relacionadas a concepção } \\
\text { ecológica }\end{array}$ & $\begin{array}{l}\text { Pigosso, Rozenfeld e McAloone (2013); Plouffe et al. } \\
\text { (2011). }\end{array}$ \\
\hline & $\begin{array}{l}\text { Incorporar tarefas de concepção ecológica para a } \\
\text { rotina diária de empregados relevantes }\end{array}$ & Pigosso, Rozenfeld e McAloone (2013). \\
\hline \multirow{10}{*}{$\begin{array}{l}\text { Produção/ } \\
\text { Manufatura }\end{array}$} & $\begin{array}{l}\text { Definir critérios ambientais para definição dos } \\
\text { equipamentos }\end{array}$ & Bonvoisin et al. (2014). \\
\hline & $\begin{array}{l}\text { Definir critérios ambientais para definição da } \\
\text { infraestrutura }\end{array}$ & Bonvoisin et al. (2014); Kurk e Eagan (2008). \\
\hline & $\begin{array}{l}\begin{array}{l}\text { Minimizar os resíduos gerados no processo } \\
\text { produtivo }\end{array} \\
\end{array}$ & $\begin{array}{l}\text { Wimmer et al. (2005); Luttropp e Lagerstedt (2006); } \\
\text { Fiksel (2009). }\end{array}$ \\
\hline & $\begin{array}{l}\text { Utilização de materiais com menor energia } \\
\text { empregada em sua fabricação ou extração }\end{array}$ & van Hemel e Cramer (2002). \\
\hline & $\begin{array}{l}\text { Utilização de menor volume de componentes no } \\
\text { produto }\end{array}$ & van Hemel e Cramer (2002); Platcheck et al. (2008b). \\
\hline & $\begin{array}{l}\text { Projetar o processo de fabricação com técnicas de } \\
\text { produção mais limpa }\end{array}$ & $\begin{array}{l}\text { van Hemel e Cramer (2002); Silva, Moraes e Machado } \\
\text { (2015). }\end{array}$ \\
\hline & Reduzir os processos produtivos & van Hemel e Cramer (2002). \\
\hline & $\begin{array}{l}\text { Reduzir a eliminação de resíduos do processo } \\
\text { produtivo }\end{array}$ & van Hemel e Cramer (2002). \\
\hline & $\begin{array}{l}\text { Reduzir uso de materiais de consumo no processo } \\
\text { de produção }\end{array}$ & van Hemel e Cramer (2002); Platcheck et al. (2008a). \\
\hline & $\begin{array}{l}\text { Utilizar materiais limpos para embalagens, de } \\
\text { baixa energia empregada e sem produtos tóxicos } \\
\text { em sua composição }\end{array}$ & van Hemel e Cramer (2002). \\
\hline \multirow{15}{*}{$\begin{array}{c}\text { Uso/ } \\
\text { Utilização }\end{array}$} & $\begin{array}{l}\text { Melhorar a logística de distribuição, minimizando a } \\
\text { necessidade de transporte }\end{array}$ & $\begin{array}{l}\text { Giannetti e Almeida (2006); Luttropp e Lagerstedt } \\
\text { (2006); González-García et al. (2011); Wimmer et al. } \\
\text { (2005); van Hemel e Cramer (2002); Alves et al. } \\
\text { (2010). }\end{array}$ \\
\hline & Aumentar a vida do produto & $\begin{array}{l}\text { Giannetti e Almeida (2006); Luttropp e Lagerstedt } \\
\text { (2006). }\end{array}$ \\
\hline & $\begin{array}{l}\text { Reduzir ou eliminar o armazenamento e emissão } \\
\text { de materiais perigosos }\end{array}$ & Giannetti e Almeida (2006). \\
\hline & Projetar produtos de forma multifuncional & $\begin{array}{l}\text { Wimmer et al. (2005); Luttropp e Lagerstedt (2006); } \\
\text { Fiksel (2009). }\end{array}$ \\
\hline & $\begin{array}{l}\text { Reaproveitar resíduos gerados durante uso do } \\
\text { produto }\end{array}$ & $\begin{array}{l}\text { Wimmer et al. (2005); Luttropp e Lagerstedt (2006); } \\
\text { Fiksel (2009); Borchardt et al. (2008). }\end{array}$ \\
\hline & $\begin{array}{l}\text { Reduzir o consumo energético na utilização do } \\
\text { produto }\end{array}$ & van Hemel e Cramer (2002). \\
\hline & $\begin{array}{l}\text { Empregar fontes de energias limpas na utilização } \\
\text { do produto }\end{array}$ & van Hemel e Cramer (2002). \\
\hline & $\begin{array}{l}\text { Utilizar poucos materiais de consumo na utilização } \\
\text { do produto }\end{array}$ & van Hemel e Cramer (2002). \\
\hline & $\begin{array}{l}\text { Utilizar materiais de consumo limpos na utilização } \\
\text { do produto }\end{array}$ & van Hemel e Cramer (2002). \\
\hline & $\begin{array}{l}\text { Obter um produto que não desperdice energia na } \\
\text { sua utilização }\end{array}$ & van Hemel e Cramer (2002). \\
\hline & Alta durabilidade e confiabilidade do produto & van Hemel e Cramer (2002). \\
\hline & $\begin{array}{l}\text { Estruturar produtos modulares ou adaptável às } \\
\text { necessidades dos clientes }\end{array}$ & van Hemel e Cramer (2002). \\
\hline & $\begin{array}{l}\text { Buscar uma forte relação do produto com seu } \\
\text { consumidor ou usuário }\end{array}$ & van Hemel e Cramer (2002). \\
\hline & Permitir o uso do produto compartilhado & van Hemel e Cramer (2002). \\
\hline & $\begin{array}{l}\text { Otimizar funcionalidades e integrar funções para o } \\
\text { produto }\end{array}$ & van Hemel e Cramer (2002). \\
\hline
\end{tabular}

Revista Produção Online. Florianópolis, SC, v. 18, n. 1, p. 310-328, 2018. 
Quadro 4 - Práticas relacionadas ao DfE categorizadas no ciclo de desenvolvimento do produto

(conclusão)

\begin{tabular}{|c|c|c|}
\hline Fase & Práticas de DfE & Literatura \\
\hline \multirow{10}{*}{$\begin{array}{l}\text { Disposição/ } \\
\text { Recuperação }\end{array}$} & $\begin{array}{l}\text { Empregar produtos recicláveis ou reutilizáveis, } \\
\text { reduzindo ou eliminando o uso de materiais } \\
\text { virgens. Empregar logística reversa. }\end{array}$ & $\begin{array}{l}\text { Giannetti e Almeida (2006); Luttropp e Lagerstedt } \\
\text { (2006); Passarini et al. (2010); van Hemel e Cramer } \\
\text { (2002); Alves et al. (2010); Boks (2006); Short et al. } \\
\text { (2012); Silva, Moraes e Machado (2015); Ardente, } \\
\text { Mathieux e Recchioni (2014). }\end{array}$ \\
\hline & Promover a reparação e modernização & Luttropp e Lagerstedt (2006). \\
\hline & $\begin{array}{l}\text { Utilizar material retornável ou reutilizável para } \\
\text { embalagem }\end{array}$ & $\begin{array}{l}\text { Roca et al. (2012); Passarini et al. (2010); Wimmer et } \\
\text { al. (2005); van Hemel e Cramer (2002). }\end{array}$ \\
\hline & Utilizar materiais renováveis & van Hemel e Cramer (2002). \\
\hline & Facilitar manutenção e reparo do produto & van Hemel e Cramer (2002). \\
\hline & Reutilizar o produto em seu fim de vida & van Hemel e Cramer (2002). \\
\hline & $\begin{array}{l}\text { Projetar um processo de remanufatura ou } \\
\text { renovação para o produto }\end{array}$ & $\begin{array}{l}\text { van Hemel e Cramer (2002); Sakundarini et al. (2015); } \\
\text { Pigosso et al. (2010). }\end{array}$ \\
\hline & $\begin{array}{l}\text { Incinerar de forma segura o produto no fim de vida } \\
\text { permitindo a recuperação de energia }\end{array}$ & van Hemel e Cramer (2002). \\
\hline & $\begin{array}{l}\text { Eliminar de forma segura, os restos do produto } \\
\text { para sua disposição }\end{array}$ & van Hemel e Cramer (2002); Borchardt et al. (2008). \\
\hline & $\begin{array}{l}\text { Desmaterialização como conceito de concepção } \\
\text { do produto }\end{array}$ & van Hemel e Cramer (2002). \\
\hline
\end{tabular}

Fonte: Elaborado pelos autores

\section{DISCUSSÃO E CONSIDERAÇÕES FINAIS}

Embora os objetivos dos trabalhos evidenciados na RBS para utilização do DfE sejam convergentes, observa-se uma grande variedade em relação as abordagens relacionados ao tema, o modo de execução, metodologia, a real necessidade de utilização e implementação.

$\mathrm{Na}$ leitura dos artigos, os objetivos estudados se assemelham para temas sobre qualidade, inovação, estatégia, melhoria ambiental, avaliação ambiental, ferramenta, integração, setor ou produto específico estudado em desenvolvimento de produto.

Muitos trabalhos relatam aplicações do DfE em áreas específicas do setor industrial, como por exemplo o químico, automobilístico, eletrônico, serviços, entre outros. Cada um contribuindo com resultados em um setor ou produto específico e de formas distintas. Também, para consolidação das práticas do DfE, os trabalhos buscam integrar os métodos ou metodologias criadas as rotinas da organização.

A Tabela 3 expõe os artigos oriundos da RBS e os relaciona aos principais temas abordados nos estudos sobre DfE e a Tabela 4 indica a frequencia da palavra no texto encontrada no corpo do artigo. 
Tabela 3 - Análise de agrupamento da RBS

\begin{tabular}{|c|c|c|c|c|c|c|c|c|}
\hline \multirow[b]{2}{*}{ Autores } & \multicolumn{8}{|c|}{ Características dos trabalhos } \\
\hline & Qualidade & Inovação & Estratégia & $\begin{array}{c}\text { Melhoria } \\
\text { ambiental }\end{array}$ & $\begin{array}{l}\text { Avaliação } \\
\text { ambiental }\end{array}$ & Ferramenta & Integração & $\begin{array}{c}\text { Setor/ } \\
\text { Produto } \\
\text { específico }\end{array}$ \\
\hline Alves et al. (2010) & & & & $\sqrt{ }$ & & & & $\sqrt{ }$ \\
\hline Andriankaja et al. (2015) & & & & $\sqrt{ }$ & $\sqrt{ }$ & $\sqrt{ }$ & & $\sqrt{ }$ \\
\hline $\begin{array}{l}\text { Arana-Landin e Heras-Saizarbitoria } \\
\text { (2011) }\end{array}$ & & & & & $\sqrt{ }$ & $\sqrt{ }$ & $\sqrt{ }$ & \\
\hline Ardente, Mathieux e Recchioni (2014) & & $\sqrt{ }$ & $\sqrt{ }$ & & & $\sqrt{ }$ & & $\sqrt{ }$ \\
\hline Birch, Hon e Short (2012) & & & $\sqrt{ }$ & $\sqrt{ }$ & & $\sqrt{ }$ & & \\
\hline Boks (2006) & & & $\sqrt{ }$ & & & $\sqrt{ }$ & $\sqrt{ }$ & \\
\hline Boks e Stevels (2007) & & & $\sqrt{ }$ & & & $\sqrt{ }$ & $\sqrt{ }$ & $\sqrt{ }$ \\
\hline Bonilla et al. (2010) & & & & $\sqrt{ }$ & $\sqrt{ }$ & $\sqrt{ }$ & $\sqrt{ }$ & $\sqrt{ }$ \\
\hline Bonvoisin et al. (2014) & & & & & $\sqrt{ }$ & $\sqrt{ }$ & $\sqrt{ }$ & $\sqrt{ }$ \\
\hline Borchardt et al. (2008) & & & $\sqrt{ }$ & $\sqrt{ }$ & & $\sqrt{ }$ & & $\sqrt{ }$ \\
\hline Borchardt et al. (2010) & & $\sqrt{ }$ & $\sqrt{ }$ & & & $\sqrt{ }$ & $\sqrt{ }$ & $\sqrt{ }$ \\
\hline Borchardt et al. (2012) & & & & & $\sqrt{ }$ & $\sqrt{ }$ & & $\sqrt{ }$ \\
\hline Bovea e Pérez-Belis (2012) & & & & & & $\sqrt{ }$ & $\sqrt{ }$ & \\
\hline Brones e de Carvalho (2015) & & $\sqrt{ }$ & & & & $\sqrt{ }$ & $\sqrt{ }$ & \\
\hline Brones, de Carvalho e Zancul (2014) & & $\sqrt{ }$ & $\sqrt{ }$ & & & $\sqrt{ }$ & $\sqrt{ }$ & $\sqrt{ }$ \\
\hline Byggeth e Hochschorner (2006) & & & $\sqrt{ }$ & & & $\sqrt{ }$ & $\sqrt{ }$ & \\
\hline González-García et al. (2011) & & & $\sqrt{ }$ & $\sqrt{ }$ & $\sqrt{ }$ & $\sqrt{ }$ & & $\sqrt{ }$ \\
\hline Gouda, Jonnalagedda, Saranga (2016) & $\sqrt{ }$ & & & & $\sqrt{ }$ & $\sqrt{ }$ & & $\sqrt{ }$ \\
\hline Hernandez et al. (2012) & & & & & & $\sqrt{ }$ & & \\
\hline Jeswiet e Hauschild (2005) & & & & & & $\sqrt{ }$ & $\sqrt{ }$ & $\sqrt{ }$ \\
\hline Kengpol e Boonkanit (2008) & & & $\sqrt{ }$ & & $\sqrt{ }$ & $\sqrt{ }$ & $\sqrt{ }$ & \\
\hline Kurk e Eagan (2008) & & $\sqrt{ }$ & & & & $\sqrt{ }$ & $\sqrt{ }$ & \\
\hline Lindahl (2006) & & & & & & $\sqrt{ }$ & $\sqrt{ }$ & $\sqrt{ }$ \\
\hline Lofthouse (2006) & & & $\sqrt{ }$ & & & $\sqrt{ }$ & $\sqrt{ }$ & $\sqrt{ }$ \\
\hline Luttropp e Lagerstedt (2006) & & & & & $\sqrt{ }$ & $\sqrt{ }$ & $\sqrt{ }$ & \\
\hline Park e Tahara (2008) & $\sqrt{ }$ & & & & & $\sqrt{ }$ & $\sqrt{ }$ & $\sqrt{ }$ \\
\hline Passarini et al. (2010) & & & & & $\sqrt{ }$ & $\sqrt{ }$ & & $\sqrt{ }$ \\
\hline Pigosso et al. (2010) & & & $\sqrt{ }$ & & & $\sqrt{ }$ & $\sqrt{ }$ & \\
\hline Pigosso, Rozenfeld e McAloone (2013) & & $\sqrt{ }$ & $\sqrt{ }$ & & & $\sqrt{ }$ & $\sqrt{ }$ & $\sqrt{ }$ \\
\hline Platcheck et al. (2008a) & & $\sqrt{ }$ & $\sqrt{ }$ & $\sqrt{ }$ & & $\sqrt{ }$ & & $\sqrt{ }$ \\
\hline Platcheck et al. (2008b) & & & $\sqrt{ }$ & & & & $\sqrt{ }$ & $\sqrt{ }$ \\
\hline Plouffe et al. (2011) & & $\sqrt{ }$ & $\sqrt{ }$ & & & & $\sqrt{ }$ & $\sqrt{ }$ \\
\hline Pochat, Bertoluci e Froelich (2007) & & & $\sqrt{ }$ & & & $\sqrt{ }$ & $\sqrt{ }$ & $\sqrt{ }$ \\
\hline Raz, Druehl e Blass (2013) & & $\sqrt{ }$ & & & & $\sqrt{ }$ & & $\sqrt{ }$ \\
\hline Roca et al. (2012) & & & & & & $\sqrt{ }$ & $\sqrt{ }$ & $\sqrt{ }$ \\
\hline Sakundarini et al. (2015) & & & & & & $\sqrt{ }$ & & $\sqrt{ }$ \\
\hline Short et al. (2012) & & & $\sqrt{ }$ & & & $\sqrt{ }$ & $\sqrt{ }$ & $\sqrt{ }$ \\
\hline Silva, Moraes e Machado (2015) & & & & & & $\sqrt{ }$ & & $\sqrt{ }$ \\
\hline Tiruta-Barna et al. (2014) & & & & & & $\sqrt{ }$ & $\sqrt{ }$ & $\sqrt{ }$ \\
\hline van Hemel e Cramer (2002) & & $\sqrt{ }$ & $\sqrt{ }$ & $\sqrt{ }$ & & & & $\sqrt{ }$ \\
\hline Zhang et al. (2011) & & & & & & $\sqrt{ }$ & $\sqrt{ }$ & $\sqrt{ }$ \\
\hline
\end{tabular}

Fonte: Elaborado pelos autores

Revista Produção Online. Florianópolis, SC, v. 18, n. 1, p. 310-328, 2018. 
Tabela 4 - Análise quantitativa da RBS

\begin{tabular}{|c|c|c|c|c|c|c|c|c|}
\hline \multirow[b]{2}{*}{ Autores } & \multicolumn{8}{|c|}{ Características dos trabalhos } \\
\hline & Qualidade & Inovação & Estratégia & $\begin{array}{l}\text { Melhoria } \\
\text { ambiental }\end{array}$ & $\begin{array}{l}\text { Avaliação } \\
\text { ambiental }\end{array}$ & Ferramenta & Integração & $\begin{array}{c}\text { Setor/Produto } \\
\text { específico }\end{array}$ \\
\hline Alves et al. (2010) & & & & 5 & & & & 17 \\
\hline Andriankaja et al. (2015) & & & & 41 & 49 & 85 & & 11 \\
\hline Arana-Landin e Heras-Saizarbitoria (2011) & & & & & 4 & 8 & 10 & \\
\hline Ardente, Mathieux e Recchioni (2014) & & 5 & 5 & & & 7 & & 69 \\
\hline Birch, Hon e Short (2012) & & & 83 & 20 & & 178 & & \\
\hline Boks (2006) & & & 10 & & & 32 & 17 & \\
\hline Boks e Stevels (2007) & & & 25 & & & 11 & 10 & 15 \\
\hline Bonilla et al. (2010) & & & & 10 & 28 & 12 & 3 & 27 \\
\hline Bonvoisin et al. (2014) & & & & & 24 & 2 & 4 & 115 \\
\hline Borchardt et al. (2008) & & & 4 & 6 & & 20 & & 16 \\
\hline Borchardt et al. (2010) & & 6 & 5 & & & 52 & 4 & 5 \\
\hline Borchardt et al. (2012) & & & & & 18 & 22 & & 5 \\
\hline Bovea e Pérez-Belis (2012) & & & & & & 75 & 49 & \\
\hline Brones e de Carvalho (2015) & & 38 & & & & 41 & 94 & \\
\hline Brones, de Carvalho e Zancul (2014) & & 13 & 13 & & & 22 & 38 & 3 \\
\hline By ggeth e Hochschorner (2006) & & & 43 & & & 158 & 4 & \\
\hline González-García et al. (2011) & & & 16 & 31 & & 1 & & 29 \\
\hline Gouda, Jonnalagedda, Saranga (2016) & 160 & & & & 6 & 3 & & 7 \\
\hline Hernandez et al. (2012) & & & & & & 88 & & \\
\hline Jeswiet e Hauschild (2005) & & & & & & 10 & 2 & 28 \\
\hline Kengpol e Boonkanit (2011) & & & 6 & & 11 & 12 & 13 & \\
\hline Kurk e Eagan (2008) & & 6 & & & & 31 & 10 & \\
\hline Lindahl (2006) & & & & & & 231 & 12 & 2 \\
\hline Lofthouse (2006) & & & 10 & & & 115 & 6 & 5 \\
\hline Luttropp e Lagerstedt (2006) & & & & & 2 & 62 & 3 & \\
\hline Park e Tahara (2008) & 46 & & & & & 9 & 7 & 22 \\
\hline Passarini et al. (2010) & & & & & 5 & 5 & & 8 \\
\hline Pigosso et al. (2010) & & & 40 & & & 25 & 8 & \\
\hline Pigosso, Rozenfeld e McAloone (2013) & & 5 & 29 & & & 58 & 15 & 16 \\
\hline Platcheck et al. (2008)a & & 5 & 5 & 7 & & 7 & & 24 \\
\hline Platcheck et al. (2008)b & & & 5 & & & & 2 & 36 \\
\hline Plouffe et al. (2011) & & 7 & 11 & & & & 6 & 15 \\
\hline Pochat, Bertoluci e Froelich (2007) & & & 19 & & & 125 & 49 & 6 \\
\hline Raz, Druehl e Blass (2013) & & 153 & & & & 4 & & 18 \\
\hline Roca et al. (2012) & & & & & & 19 & 14 & 17 \\
\hline Sakundarini et al. (2015) & & & & & & 38 & & 59 \\
\hline Short et al. (2012) & & & 12 & & & 10 & 10 & 12 \\
\hline Silva, Moraes e Machado (2015) & & & & & & 16 & & 1 \\
\hline Tiruta-Barna et al. (2014) & & & & & & 13 & 6 & 1 \\
\hline van Hemel e Cramer (2002) & & 24 & 23 & 49 & & & & 54 \\
\hline Zhang et al. (2011) & & & & & & 4 & 4 & 14 \\
\hline
\end{tabular}

Fonte: Elaborado pelos autores

Esta pesquisa sistematiza uma forma de revisão bibliográfica para o DfE, onde é possível verificar os principais pesquisadores no tema e compreender a contribuição específica de cada um sobre DfE. Observa-se que a maioria dos autores o consideram 
uma ferramenta para desenvolvimento do produto que possibilita a integração das etapas de desenvolvimento com as de fabricação. Um aspecto curioso observado é que, embora por definição, o DfE vise a melhoria ambiental esse tema não é citado massivamente nos artigos analisados.

O estudo realizado permitiu classificar a bibliografia sobre o tema, de acordo com as etapas de do ciclo de desenvolvimento do produto, e explicitar, de forma sumarizada, as práticas do DfE em cada uma dessas etapas. A identificação das práticas pode ser um ponto a ser explorado em pesquisas futuras, onde novos estudos, mais recentes, contribuição para ampliar essa base de práticas aqui apresentadas.

A grande limitação desse trabalho está relacionada a sua abrangência uma vez que somente duas bases de dados foram analisadas os critérios de inclusão e exclusão.

A RBS desta pesquisa contribui para pesquisas futuras em aplicações de práticas de DfE específicas como trabalhos que exploram o tema.

\section{REFERÊNCIAS}

ALVES, C. et al. Ecodesign of automotive components making use of natural jute fiber composites. Journal of Cleaner Production, v. 18, n. 4, p. 313-327, 2010. https://doi.org/10.1016/j.jclepro.2009.10.022

ANDRIANKAJA, $\mathrm{H}$. et al. A method to ecodesign structural parts in the transport sector based on product life cycle management. Journal of Cleaner Production, v. 94, p. 165176, 2015. https://doi.org/10.1016/j.jclepro.2015.02.026

ARANA-LANDIN, G.; HERAS-SAIZARBITORIA, I. Paving the way for the ISO 14006 ecodesign standard: An exploratory study in Spanish companies. Journal of Cleaner Production, v. 19, n. 9-10, p. 1007-1015, 2011. https://doi.org/10.1016/j.jclepro.2011.01.013

ARDENTE, F.; MATHIEUX, F.; RECCHIONI, M. Recycling of electronic displays: Analysis of pre-processing and potential ecodesign improvements. Resources, Conservation and Recycling, v. 92, p. 158-171, 2014. https://doi.org/10.1016/j.resconrec.2014.09.005

BAUMANN, H.; BOONS, F.; BRAGD, A. Mapping the green product development field: engineering, policy and business perspectives. Journal of Cleaner Production, v. 10, $p$. 409-425, 2002. https://doi.org/10.1016/S0959-6526(02)00015-X

BIOLCHINI, J. et al. Systematic review in software engineering. Technical Report. Systems Engineering and Computer Science Department. COPPEUFRJ, Rio de Janeiro, 2005.

BIRCH, A.; HON, K. K. B.; SHORT, T. Structure and output mechanisms in Design for 
Environment (DfE) tools. Journal of Cleaner Production, v. 35, p. 50-58, 2012. https://doi.org/10.1016/j.jclepro.2012.05.029

BOKS, C. The soft side of ecodesign. Journal of Cleaner Production, v. 14, n. 15-16, p. 1346-1356, 2006. https://doi.org/10.1016/j.jclepro.2005.11.015

BOKS, C.; STEVELS, A. Essential perspectives for design for environment. Experiences from the electronics industry. International Journal of Production Research, v. 45, n. 18, p. 4021-4039, 2007. http://dx.doi.org/10.1080/00207540701439909

BONILLA, S. H. et al. Emergy as a tool for Ecodesign: evaluating materials selection for beverage packages in Brazil. Journal of Cleaner Production, v. 18, n. 1, p. 32-43, 2010. https://doi.org/10.1016/j.jclepro.2009.03.019

BONVOISIN, J. et al. An integrated method for environmental assessment and ecodesign of ICT-based optimization services. Journal of Cleaner Production, v. 68, p. 144-154, 2014. https://doi.org/10.1016/j.jclepro.2014.01.003

BORCHARDT, M. et al. Considerações sobre ecodesign: um estudo de caso na indústria eletrônica automotiva. Ambiente \& sociedade, v. 11, n. 2, p. 341-353, 2008.

BORCHARDT, M. et al. Reprojeto do contraforte: um caso de aplicação do ecodesign em manufatura calçadista. Produção, v. 20, n. 3, p. 392-403, 2010. https://doi.org/10.1590/S0103-65132010005000006

BORCHARDT, M. et al. Avaliação da presença de práticas do Design for Environment (DfE) no desenvolvimento de produto de uma empresa da indústria química. Produção, v. 22, n. 1, p. 58-69, jan/fev. 2012. doi: 10.1590/S0103-65132012005000001

BOVEA, M. D.; PÉREZ-BELIS, V. A taxonomy of ecodesign tools for integrating environmental requirements into the product design process. Journal of Cleaner

Production, v. 20, n. 1, p. 61-71, 2012. https://doi.org/10.1016/j.jclepro.2011.07.012

BRONES, F.; DE CARVALHO, M. From 50 to 1: Integrating literature toward a systemic ecodesign model. Journal of Cleaner Production, v. 96, p. 44-47, 2015.

https://doi.org/10.1016/j.jclepro.2014.07.036

BRONES, F.; DE CARVALHO, M. M.; ZANCUL, E. Ecodesign in project management: A missing link for the integration of sustainability in product development? Journal of Cleaner Production, v. 80, p. 106-118, 2014. https://doi.org/10.1016/j.jclepro.2014.05.088

BYGGETH, S.; HOCHSCHORNER, E. Handling trade-offs in Ecodesign tools for sustainable product development and procurement. Journal of Cleaner Production, v. 14, n. 15-16, p. 1420-1430, 2006. https://doi.org/10.1016/j.jclepro.2005.03.024

CHARTER M.; TISCHNER U. Sustainable solutions. Sheffield, UK: Greenleaf Publishing, 2001.

CHIANG, T. A.; ROY, R. An intelligent benchmark-based design for environment system for derivative eletronic product development. Computers in Industry, v. 63, p. 913-929, 2012. https://doi.org/10.1016/i.compind.2012.08.014

CONFORTO, E. C.; AMARAL, D. C.; SILVA, S. L. DA. Roteiro para revisão bibliográfica sistemática: aplicação no desenvolvimento de produtos e gerenciamento de projetos. $8^{\circ}$ 
Congresso Brasileiro de Gestão de Desenvolvimento de Produto - CNGDP 2011, n. 1998, p. 1-12, 2011.

COOK, D. J.; MULROW, C. D.; HAYNES, R. B. Systematic reviews: synthesis of best evidence for clinical decisions. Annals of Internal Medicine, v.126, n.5, p. 376-380, 1997. DOI: 10.7326/0003-4819-126-5-199703010-00006

COOPER, H. Synthesizing Research. Thousand Oaks: Sage, 1998.

DANGELICO, R. M.; PONTRANDOLFO, P. From green product definitions and classifications to the Green Option Matrix. Journal of Cleaner Production, v. 18, n. 16-17, p. 1608-1628, nov. 2010. https://doi.org/10.1016/j.jclepro.2010.07.007

FIKSEL, J. Ingeniería de diseño medioambiental. DEF: desarrollo integral de productos y processos ecoeficientes. Madrid: McGrawHill Book, 1997.

FIKSEL, J. Design for Environment: A Guide to Sustainable Product Development. 2.ed. New York: McGraw Hill, 2009.

GIANNETTI, B. F.; ALMEIDA, C. M. V. B. Ecologia industrial: conceitos, ferramentas e aplicações. São Paulo: Blucher, 2006.

GONZÁLEZ-GARCÍA, S. et al. Assessing the global warming potential of wooden products from the furniture sector to improve their ecodesign. Science of the Total Environment, v. 410-411, p. 16-25, 2011. https://doi.org/10.1016/i.scitotenv.2011.09.059

GOUDA, S. K.; JONNALAGEDDA, S.; SARANGA, H. Design for the environment: Impact of regulatory policies on product development. European Journal of Operational Research, v. 248 , n. 2, p. 558-570, 2016. https://doi.org/10.1016/j.ejor.2015.07.043

HERNANDEZ, N. V. et al. Development of an expert system to aid engineers in the selection of design for environment methods and tools. Expert Systems with Applications, v. 39, n. 10, p. 9543-9553, ago. 2012. https://doi.org/10.1016/j.eswa.2012.02.098

HOUE, R.; GRABOT, B. Assessing the compliance of a product with an eco- label: from standards to constraints. International Journal of Production Economics, v. 121, p. $21-$ 38, 2009. https://doi.org/10.1016/j.ijpe.2008.03.014

JESWIET, J.; HAUSCHILD, M. EcoDesign and future environmental impacts. Materials \& Design, v. 26, n. 7, p. 629-634, 2005. https://doi.org/10.1016/j.matdes.2004.08.016

KENGPOL, A.; BOONKANIT, P. The decision support framework for developing Ecodesign at conceptual phase based upon ISO/TR 14062. International Journal of Production Economics, v. 131, n. 1, p. 4-14, 2011. https://doi.org/10.1016/j.ijpe.2010.10.006

KURK, F.; EAGAN, P. The value of adding design-for-the-environment to pollution prevention assistance options. Journal of Cleaner Production, v. 16, n. 6, p. 722-726, 2008. https://doi.org/10.1016/j.jclepro.2007.02.022

LEWIS, H. et al. Design+environment: a global guide to designing greener goods. sheffield, UK: Greenleaf Publishing Limited, 2001. 
LINDAHL, M. Engineering designers' experience of design for environment methods and tools e Requirement definitions from an interview study. Journal of Cleaner Production, $v$. 14, n. 5, p. 487-496, 2006. https://doi.org/10.1016/j.jclepro.2005.02.003

LOFTHOUSE, V. A. Ecodesign tools for designers: defining the requirements. Journal of Cleaner Production, v. 14, n. 15-16, p. 1386-1395, 2006.

https://doi.org/10.1016/j.jclepro.2005.11.013

LUTTROPP, C.; LAGERSTEDT, J. EcoDesign and the ten golden rules: generic advice for merging environmental aspects into product development. Journal of Cleaner Production, v. 14, n. 15-16, p. 1396-1408, 2006. https://doi.org/10.1016/j.jclepro.2005.11.022

MATOS, S.; HALL, J. Integrating sustainable development in the supply chain: the case of sustainable development in the oil and gas and agricultural biotechnology. Journal of Operations Managment, v. 25, n. 6, p. 1083-1102, nov. 2007. https://doi.org/10.1016/j.jom.2007.01.013

MIETTINEM, P.; HAMALAINEN, R. P. How to benefit from decision analysis in environmental life cycle assessment (LCA). European Journal of of Operational Research, v. 102, n. 2, p. 279-294, 1997. https://doi.org/10.1016/S0377-2217(97)00109-4

MONTABON, F.; SROUFE, R.; NARASIMHAN, R. An examination of corporate reporting, environmental management practices and firm performance. Journal of Operations Management, v. 25, n. 5, p. 998-1014, 2007. https://doi.org/10.1016/i.jom.2006.10.003

PARK, P. J.; TAHARA, K. Quantifying producer and consumer-based eco-efficiencies for the identification of key ecodesign issues. Journal of Cleaner Production, v. 16, n. 1, p. 95104, 2008. https://doi.org/10.1016/j.jclepro.2006.11.003

PASSARINI, F. et al. Assessment of Ecodesign potential in reaching new recycling targets. Resources, Conservation and Recycling, v. 54, n. 12, p. 1128-1134, 2010. https://doi.org/10.1016/j.resconrec.2010.03.006

PIGOSSO, D. C. A. et al. Ecodesign methods focused on remanufacturing. Journal of Cleaner Production, v. 18, n. 1, p. 21-31, 2010.

https://doi.org/10.1016/j.jclepro.2009.09.005

PIGOSSO, D. C. A.; ROZENFELD, H.; MCALOONE, T. C. Ecodesign maturity model: A management framework to support ecodesign implementation into manufacturing companies. Journal of Cleaner Production, v. 59, p. 160-173, 2013. https://doi.org/10.1016/j.jclepro.2013.06.040

PLATCHECK, E. R. et al. Methodology of ecodesign for the development of more sustainable electro-electronic equipments. Journal of Cleaner Production, v. 16, n. 1, p. 75-86, 2008a. https://doi.org/10.1016/j.jclepro.2006.10.006

PLATCHECK, E. R. et al. EcoDesign: case of a mini compressor re-design. Journal of Cleaner Production, v. 16, n. 14, p. 1526-1535, 2008b. https://doi.org/10.1016/j.jclepro.2007.09.004

PLOUFFE, S. et al. Economic benefits tied to ecodesign. Journal of Cleaner Production, v. 19, n. 6-7, p. 573-579, 2011. https://doi.org/10.1016/j.jclepro.2010.12.003 
POCHAT, S.; BERTOLUCI, G.; FROELICH, D. Integrating ecodesign by conducting changes in SMEs. Journal of Cleaner Production, v. 15, n. 7, p. 671-680, 2007.

https://doi.org/10.1016/j.jclepro.2006.01.004

RAZ, G.; DRUEHL, C. T.; BLASS, V. Design for the Environment: Life-Cycle Approach Using a Newsvendor Model. Production and operations management, v. 22, n. 4, p. 940-957,

2013. https://doi.org/10.1111/poms.12011

ROCA, E. et al. Application of fuzzy logic for the integration of environmental criteria in ecodesign. Expert Systems with Applications, v. 39, n. 4, p. 4427-4431, 2012. https://doi.org/10.1016/j.eswa.2011.09.148

SAKUNDARINI, N. et al. Design for environment and design for disassembly practices in Malaysia: A practitioner's perspectives. Journal of Cleaner Production, v. 108, p. 331-342, 2015. https://doi.org/10.1016/j.jclepro.2015.06.033

SHORT, T. et al. Manufacturing, sustainability, ecodesign and risk: lessons learned from a study of Swedish and English companies. Journal of Cleaner Production, v. 37, p. 342352, 2012. https://doi.org/10.1016/j.jclepro.2012.07.037

SILVA, A. L. E.; MORAES, J. A. R.; MACHADO, E. L. Proposta de produção mais limpa voltada às práticas de ecodesign e logística reversa. Engenharia Sanitária e Ambiental, v. 20, n. 1, p. 29-37, 2015. http://dx.doi.org/10.1590/S1413-41522015020000087843

SROUFE, R. Effect of environmental management systems on environmental management practices and operations. Production and Operations Management Journal, v. 12, n. 3, p. 416-431, 2003. https://doi.org/10.1111/j.1937-5956.2003.tb00212.x

TIRUTA-BARNA, L. et al. Formalization of a technical procedure for process ecodesign dedicated to drinking water treatment plants. Journal of Cleaner Production, v. 68, p. 1624, 2014. https://doi.org/10.1016/j.jclepro.2014.01.011

VAN HEMEL, C.; CRAMER, J. Barriers and stimuli for ecodesign in SMEs. Journal of Cleaner Production, v. 10, p. 439-453, 2002. https://doi.org/10.1016/S0959-6526(02)00013$\underline{6}$

WIMMER, W. et al. Product innovation through ecodesign. International Journal of Sustainable Design, v. 1, p. 75-92, 2005. http://dx.doi.org/10.1504/IJSDES.2008.017058

ZHANG, L. et al. Development and analysis of design for environment oriented design parameters. Journal of Cleaner Production, v. 19, n. 15, p. 1723-1733, out. 2011. https://doi.org/10.1016/j.jclepro.2011.06.012

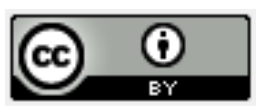

Artigo recebido em 03/03/2017 e aceito para publicação em 18/04/2017 DOI: http://dx.doi.org/10.14488/1676-1901.v18i1.2784 